\title{
Relationship between cerebral blood flow reduction patterns on scintigraphy and nonmotor symptoms in new-onset Lewy
} body disease

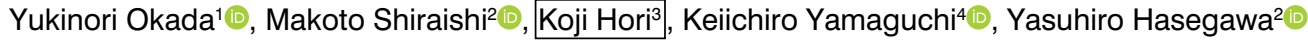 \\ ${ }^{1}$ Department of Medical Radiology, St. Marianna University School of Medicine, Kawasaki, Japan \\ 'Department of Internal Medicine and Brain Neurology, St. Marianna University School of Medicine, Kawasaki, Japan \\ ${ }^{3}$ Department of Neuropsychiatry, St. Marianna University School of Medicine, Kawasaki, Japan \\ ${ }^{4}$ Department of Proton Therapy and Tumor Imaging, St. Marianna University School of Medicine, Kawasaki, Japan
}

[Received 27 VI 2021; Accepted 31 XII 2021]

\begin{abstract}
Background: This study aimed to investigate the relationship between patterns of reduced cerebral blood flow (CBF) evaluated by means of ${ }^{123} \mid-\mathrm{N}$-isopropyl-p-iodoamphetamine ([123|]IMP) scintigraphy and nonmotor symptoms in new-onset Lewy body disease (Parkinson's disease and dementia with Lewy bodies [DLB]).

Material and methods: Twenty-four patients diagnosed with new-onset Parkinson's disease or DLB underwent [123] IMP CBF scintigraphy at St. Marianna Medical University Hospital between January 1, 2010, and March 30, 2018. The reductions in CBF in various brain regions were analyzed using the three-dimensional stereotactic surface projection method and were compared to standard database values, yielding extent values (\%). The extent values were evaluated in relation to the presence/absence of motor or nonmotor symptoms such as visual hallucinations, auditory hallucinations, delirium, depression, delusions, and dementia.
\end{abstract}

Results: The extent value was $100 \%$ in the angular, supramarginal, and lingual gyri; $95 \%$ in the orbital gyri; and $92.6 \%$ in the fusiform gyri. The extent value in patients without hallucinations and those with visual hallucinations was $41.2 \%$ and $54.3 \%$, respectively, in the frontal lobe $(p=0.02)$ and $33.3 \%$ and $51.0 \%$, respectively, in the medial prefrontal gyri $(p=0.02)$. Age-adjusted multivariate analysis showed that extent values in the frontal lobe were associated with visual hallucinations (odds ratio: $1.09,95 \%$ confidence interval 1.00-1.18, $p=0.04)$.

Conclusions: The above results show that the CBF is reduced in several areas of the cerebral cortex and suggest an association between reduced blood flow in the frontal lobe and the appearance of visual hallucinations in patients with new-onset DLB.

KEY words: hallucinations; dementia with Lewy bodies; radionuclide imaging

Nucl Med Rev 2022; 25, 1: 18-24

\section{Introduction}

Lewy body disease, an umbrella term encompassing Parkinson's disease (PD) and dementia with Lewy bodies (DLB), is reported to occur in about 1 in 1000 adults [1]. This disease

Correspondence to: Yukinori Okada, Department of Medical Radiology, St. Marianna University School of Medicine, 2-16-1 Sugao, Kawasaki City, Japan, phone: +81449778111; e-mail: igaueno512@yahoo.co.jp develops owing to the deposition of alpha-synuclein aggregates, called Lewy bodies, in the brain, resulting in neuronal damage. In $\mathrm{PD}$, Lewy body deposits are limited to the brain stem, whereas in DLB, the deposits extend to the cerebral cortex, subcortex, and peripheral neurons [2,3]. Between $8 \%$ and $40 \%$ of patients with PD experience visual hallucinations during the course of the disease [4].

${ }^{18}$ Fluorine-labeled fluorodeoxyglucose positron emission tomography $\left(\left[{ }^{18} \mathrm{~F}\right] \mathrm{FDG} \mathrm{PET}\right)$ is widely used to diagnose functional cognitive impairment and has been reported to be useful for the diagnosis of visual hallucinations associated with DLB [5]. However,

This article is available in open access under Creative Common Attribution-Non-Commercial-No Derivatives 4.0 International (CC BY-NC-ND 4.0) license, allowing to download articles and share them with others as long as they credit the authors and the publisher, but without permission to change them in any way or use them commercially. 
in Japan, $\left[{ }^{18} \mathrm{~F}\right] \mathrm{FDG}$ PET is not routinely used to diagnose Lewy body disease or Alzheimer's disease. Instead, these are commonly diagnosed by means of cerebral blood flow (CBF) scintigraphy [6]. The extent of $\mathrm{CBF}$ reduction associated with $\mathrm{PD}$ has been shown to differ between Hoehn and Yahr (H\&Y) stages [7]. In addition, both patients with Alzheimer's disease and those with DLB exhibit reduced $\left[{ }^{18} \mathrm{~F}\right] \mathrm{FDG}$ metabolism in the temporoparietal junction, posterior cingulate gyri, precuneus, and medial occipital lobe [8]. The cingulate island sign is useful for distinguishing DLB from Alzheimer's disease on $\left[{ }^{18} \mathrm{~F}\right] \mathrm{FDG}$ PET [6] and has been shown to be an excellent discriminator between Alzheimer's disease and DLB when assessed using ${ }^{99 m}$ Tc-L-ethyl cysteinate dimer CBF scintigraphy and the easy Z-score imaging system (eZIS; FUJIFILM Toyama Chemical Co., Ltd, Tokyo) analysis software [9].

$\left[{ }^{123}\right.$ ] $]$ IMP scintigraphy is used for CBF evaluation. Three-dimensional stereotactic surface projection (3D-SSP) is commonly used to improve the diagnostic accuracy of PET and single-photon emission computed tomography (SPECT) [10]. In a previous study incorporating 3D-SSP, CBF values obtained from [ [23]]IMP scintigraphy were compared statistically to those from a normal database and those from a CT-based attenuation correction normal database [11]. The cerebral metabolic changes associated with PD and those associated with Alzheimer's disease can be distinguished by means of 3D-SSP [12]. However, few studies have examined the relationship between nonmotor symptoms and CBF in patients with new-onset Lewy body disease. In addition, reports on CBF reductions related to dementia associated with PD and DLB are scarce. Moreover, there have been few attempts to quantify CBF in patients with $P D$ dementia and in those with DLB.

In this study, we examined the patterns of CBF reduction in patients with new-onset Lewy body disease by using [ ${ }^{123}$ ] IMP scintigraphy and compared the findings based on the presence or absence of motor and nonmotor symptoms.

\section{Material and methods}

\section{Ethical considerations}

This retrospective study was approved by the Ethics Committee of St. Marianna Medical University Hospital, Kawasaki, Japan (approval no. 4019). Patients were given the opportunity to opt out through the hospital website and in the hospital.

\section{Patients}

Image interpretation terminals and electronic medical records were searched for patients diagnosed with new-onset PD or new-onset DLB who underwent [123|]IMP CBF scintigraphy at St. Marianna Medical University Hospital between January 1, 2010, and March 30, 2018.

All patients were evaluated by neurologists and psychiatrists. PD was diagnosed on the basis of the UK Parkinson's Disease Society's Brain Bank diagnostic criteria [13], and DLB was diagnosed according to the clinical diagnostic criteria proposed by McKeith et al. [14]. Nonmotor symptoms were diagnosed on the basis of the Unified Parkinson's Disease Rating Scale, Part 1, items 1, 2, and 3 (with a score above 1 indicating the presence of symptoms) [15].

At the time of ${ }^{\left[{ }^{123} \mid\right]}$ IMP CBF scintigraphy, 16 of the 24 patients included in the study were being treated with levodopa and carbidopa hydrate in combination ( $n=9)$, levodopa $(n=2)$, ropinirole hydrochloride $(n=2)$, selegiline $(n=1)$, or donepezil hydrochloride $(\mathrm{n}=2)$.

\section{CBF scintigraphy and 3D-SSP imaging}

Immediately after injection of $111 \mathrm{MBq}\left[{ }^{123}\right]$ IMP (Nihon Medi-Physics Co., Tokyo, Japan), SPECT was performed with a triple detector system (GCA-9300, Canon Medical Systems, Ohtawara, Japan) equipped with a fan-beam collimator. Image reconstruction was performed with a ramp filter and Fourier back projection. The $\left[{ }^{123} \mid\right]$ IMP scintigraphy CBF values were compared to normal CBF values derived from 3D-SSP images. The 3D-SSP database was derived from 34 volunteers ( 17 men and 17 women, aged $61.7 \pm 8.0$ years)

We calculated the $\mathrm{CBF}$ reductions in various brain regions by comparing $\mathrm{CBF}$ values obtained in our patients against normal reference values. The CBF reduction was calculated as follows: number of pixels exceeding the threshold number in a region/number of pixels in that region. The threshold values were considered abnormal when the Z-score (Z-score $=$ [normal group mean voxel — case voxel]/[normal group standard deviation]) was greater than 2 , which is quite close to the commonly used threshold value of 1.96 [16]. When differences were observed between the left and right cerebral hemispheres, the larger value was selected for analysis.

\section{Data and statistical analyses}

For the purpose of the study, we compared the extent values of each brain region between patients with PD and those with DLB. We also compared the extent values within each brain region in relation to the presence vs absence of nonmotor symptoms, such as visual hallucinations, auditory hallucinations, delirium, depression, delusions, and dementia, and in relation to the presence vs absence of motor symptoms, such as rigidity, tremors, abnormal postural reflex, hypokinesia, mask-like facies, and short-stepped gait.

Median values were calculated, and between-group differences were analyzed by means of a Mann-Whitney U test. Univariate and multivariate analyses were conducted to identify factors associated with visual hallucinations. All analyses were performed with EZR, a statistical analysis software program developed at Jichi Medical University's Saitama Medical Center (Jichi Medical University's Omiya Hospital). P-values $<0.05$ were considered statistically significant.

\section{Results}

Clinical characteristics of the study patients are summarized in Table 1. The total group comprised 14 men and 10 women, aged 41-86 years (median, 75 years), with new-onset PD $(n=15)$ or new-onset DLB $(n=9)$. Patient distribution according to the H\&Y stages was as follows: stage I, $\mathrm{n}=3$; stage $\mathrm{II}, \mathrm{n}=6$; stage III, $\mathrm{n}=11$; and stage IV, $\mathrm{n}=4$. Motor symptoms were noted in the following proportions of the study cohort: rigidity, $58.8 \%$; tremors, $50.0 \%$; abnormal postural reflex, $12.5 \%$; hypokinesia, $8.3 \%$; masklike facies, $54.1 \%$; and short-stepped gait, 45.8\%. Nonmotor symptoms were noted in the following proportions of the study cohort: visual hallucination, 25\%; auditory hallucinations, 4.1\%; delirium, $12.3 \%$; depression, $16.4 \% 1$ patient had a past history and received therapy; delusions, $8.2 \%$; and dementia, $71 \%$. 
Table 1. Patient clinical characteristics $(n=24)$

$\begin{array}{lc}\text { Age (median [range]; years) } & 75[41-86] \\ \text { Sex ratio (male/female) } & 14 / 10 \\ \text { Parkinson disease/Lewy body dementia } & 15 / 9 \\ \text { Hoehn and Yahr stage I/II//II//V } & 3 / 6 / 11 / 4 \\ \text { Motor symptoms } & \\ \text { Rigidity (\%) } & 58.8 \\ \text { Tremors (\%) } & 50.0 \\ \text { Abnormal postural reflex (\%) } & 12.5 \\ \text { Hypokinesia (\%) } & 8.3 \\ \text { Mask-like facies (\%) } & 54.1 \\ \text { Short-stepped gait (\%) } & 45.8 \\ \text { Nonmotor symptoms } & \\ \text { Visual hallucinations (\%) } & 25 \\ \text { Auditory hallucinations (\%) } & 4.1 \\ \text { Delirium (\%) } & 12.3 \\ \text { Depression (\%) (1 patient had a history of } & 16.4 \\ \text { depression) } & \\ \text { Delusions (\%) } & 8.2 \\ \text { Dementia (\%) } & 71 \\ \text { Data are presented as numbers or percentages, unless otherwise indicated }\end{array}$

The extent values per lobe and per respective gyri for the total patient group are summarized in Table 2. Of the four cerebral lobes, the occipital lobe showed the greatest CBF reduction (at $89.6 \pm 18.1 \%)$. Among the cerebral gyri, the angular ( $98.9 \pm 4.23 \%)$, supramarginal $(98.8 \pm 4.00 \%)$, and lingual gyri (91.9 $\pm 15 \%)$ showed the greatest extent values, with all three values reflective of a mean decrease in the extent values of $90 \%$ or more. The distribution of notable extent values in the occipital lobe in patients with $\mathrm{PD}$ and those with DLB is shown in Figure 1. The extent value in the occipital lobe was significantly greater in patients with DLB than in those with PD (99.9\% vs 91.5\%; $p=0.01$; Figure 1 - left panel). Additionally, compared to patients with PD, patients with DLB had significantly greater extent values in the posterior cingulate cortex (77.3\% vs 32.7\%; $p<0.01$; Figure 1 - middle panel) and precuneus (74.8\% vs 59.5\%; $p=0.03$; Figure 1 - right panel).
There were no significant differences in the extent values in other brain regions according to the presence or absence of the following nonmotor symptoms: auditory hallucinations, delirium, depression, delusions, and dementia.

The distribution of notable extent values in patients with and without visual hallucinations is shown in Figure 2. The prevalence of visual hallucinations was $0 \%$ among patients with PD (0/15) and $66.7 \%$ among those with DLB (6/9). In the frontal lobe, the extent value was $41.2 \%$ in patients without visual hallucinations and $54.3 \%$ in patients with visual hallucinations $(p=0.02$; Figure $2-$ left panel). In the medial frontal gyrus, the extent value was 33.3\% in patients without visual hallucinations and $51.0 \%$ in patients with visual hallucinations ( $p=0.02$; Figure 2 - right panel). More specifically, in the right medial frontal gyrus, the extent value was $27.2 \%$ and $48.4 \%$ in patients without and with visual hallucinations, respectively $(p=0.01)$; in the left medial frontal gyrus, the extent value was $28.1 \%$ and $51.0 \%$ in patients without and with visual hallucinations, respectively ( $p=0.01$ ). No significant difference in the extent values in any other brain region was found between patients without and with visual hallucinations.

In quantifying the relationship between extent values and visual hallucinations, both univariate and age-adjusted multivariate analyses (stepwise regression) showed reduced CBF in the frontal lobe to be statistically significant (odds ratio [OR]: 1.09, 95\% confidence interval [CI] 1.00-1.18; $p=0.04$; Table 3). Age, sex, H\&Y stage, presence of PD vs that of DLB, and use of cholinesterase inhibitors or anti-Parkinson agents were not associated with visual hallucinations. Moreover, both univariate and age-adjusted multivariate analyses (stepwise regression) showed reduced CBF in the right, left, or both frontal lobes specifically and in the medial frontal gyri (right, left, or both specifically), with that in the right frontal lobe being statistically significant (OR: 1.09, 95\% Cl 1.01-1.18, $\mathrm{p}=0.04$; Table 4).

\section{Discussion}

In this study, we examined the patterns of CBF reduction in patients with new-onset Lewy body disease and compared the findings based on the presence or absence of motor and nonmotor
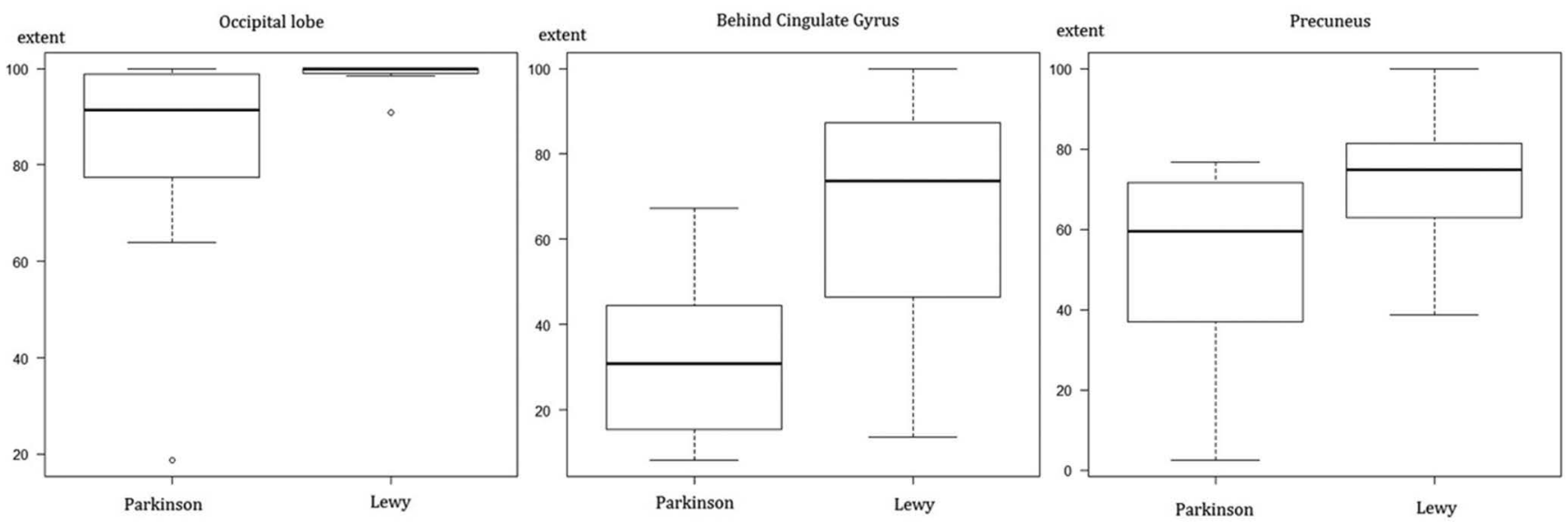

Figure 1. Box and whisker plots of the extent values for patients with Parkinson disease and patients with Lewy body disease 

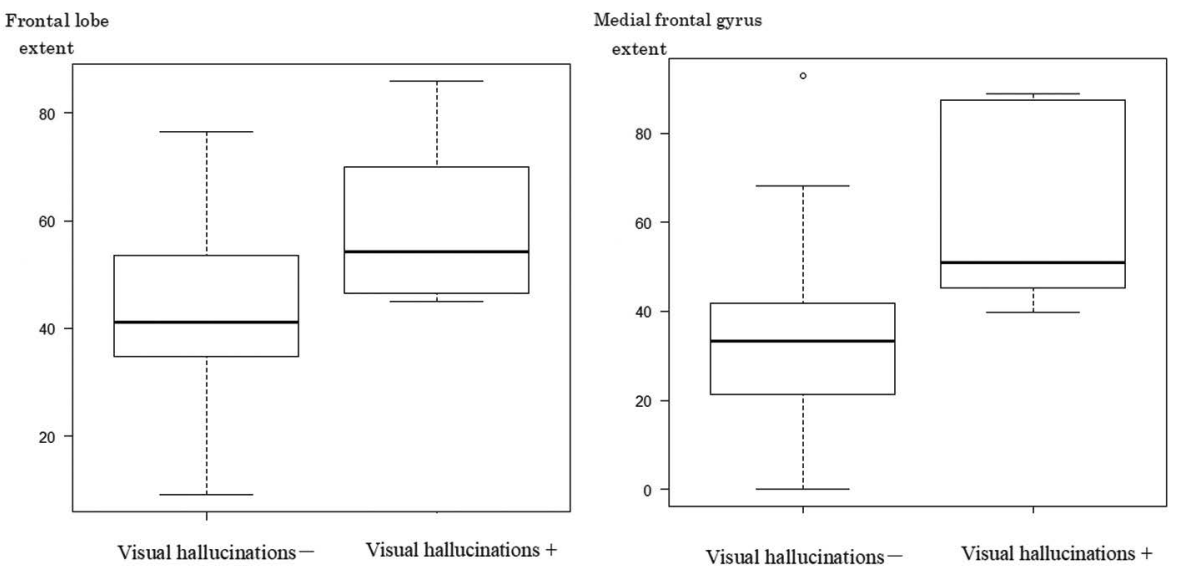

Figure 2. Box and whisker plots of the extent values in the frontal lobe and medial frontal lobe for patients with and without visual hallucinations

Table 2. Extent values per lobe and respective gyri

\begin{tabular}{lc|} 
& Extent Value (\%) \\
\hline Frontal lobe & $45.4 \pm 18.1$ \\
Orbital gyri & $71.8 \pm 39.1$ \\
Gyri recti & $51.9 \pm 33.3$ \\
Parietal lobe & $64.3 \pm 17.4$ \\
Angular gyri & $98.9 \pm 4.23$ \\
Precuneus & $58.6 \pm 25.6$ \\
Supramarginal gyri & $98.8 \pm 4.00$ \\
Temporal lobe & $80.2 \pm 18.0$ \\
Fusiform gyri & $84.5 \pm 16.0$ \\
Occipital lobe & $89.6 \pm 18.1$ \\
Lingual gyri & $91.9 \pm 15.1$ \\
Anterior cingulate gyri & $35.2 \pm 29.6$ \\
Posterior cingulate gyri & $47.1 \pm 26.8$ \\
\hline Data are presented as mean + standard deviation &
\end{tabular}

symptoms. Our results showed that the CBF is reduced in several areas of the cerebral cortex in these patients. Furthermore, our findings suggest an association between reduced blood flow in the frontal lobe and the appearance of visual hallucinations in patients with new-onset Lewy body disease.

There have been several reports on CBF scintigraphy in patients with PD and those with DLB. Patients with H\&Y stages I-II disease were found to exhibit reduced CBF in the gray matter (reduced diffusion), hippocampus, and right temporal lobe inferior horn, whereas those with H\&Y stages III-IV disease were found to exhibit reduced $\mathrm{CBF}$ in the cerebrum overall and relatively increased $\mathrm{CBF}$ in the left and right thalamus, globus pallidus, hippocampus, dentate nucleus, and right insular cortex [7]. DLB has been shown to be characterized by reduced $\left[{ }^{18} \mathrm{~F}\right] \mathrm{FDG}$ metabolism in the temporoparietooccipital lobe [8]. Patients with DLB, compared to patients with Alzheimer's disease, exhibit more prominently reduced $\mathrm{CBF}$ in the two occipital lobes and in the left temporal lobe [17]. Patients with DLB exhibit what appears to be decreased CBF in the

Table 3. Results of univariate and multivariate analyses (stepwise regression) for identification of factors associated with visual hallucinations

\begin{tabular}{|c|c|c|c|c|c|c|}
\hline \multirow[b]{2}{*}{ Factor } & \multicolumn{3}{|c|}{ Univariate Analysis } & \multicolumn{3}{|c|}{ Multivariate Analysis } \\
\hline & OR & $95 \% \mathrm{Cl}$ & $p$ value & OR & $95 \% \mathrm{Cl}$ & $p$ value \\
\hline Age & 1.32 & $1.02-1.72$ & 0.03 & 1.47 & $0.99-2.19$ & 0.06 \\
\hline Sex & 1.57 & $0.25-10.1$ & 0.63 & & & \\
\hline H\&Y stage (0-II vs III-IV) & 0.50 & $0.08-3.27$ & 0.47 & & & \\
\hline Use of a cholinesterase inhibitor or anti-Parkinson agent & 0.14 & $0.02-1.09$ & 0.06 & & & \\
\hline PD or DLB & NA & NA & NA & & & \\
\hline Frontal lobe & 1.07 & $0.99-1.15$ & 0.06 & 1.09 & $1.00-1.18$ & 0.04 \\
\hline Parietal lobe & 1.07 & $0.99-1.16$ & 0.08 & & & \\
\hline Temporal lobe & 0.97 & $0.93-1.02$ & 0.27 & & & \\
\hline Occipital lobe & 1.68 & $0.68-4.13$ & 0.26 & & & \\
\hline
\end{tabular}

$\mathrm{Cl}$ — confidence interval; H\&Y — Hoehn and Yahr; LBD — Lewy body disease; NA — not available; OR — odds ratio; PD — Parkinson's disease 
Table 4. Results of the univariate and multivariate analyses (stepwise regression) for visual hallucinations related to the frontal lobe (right, left, both) and medial frontal gyri (right, left, both)

\begin{tabular}{|c|c|c|c|c|c|c|}
\hline \multirow[b]{2}{*}{ Factor } & \multicolumn{3}{|c|}{ Univariate analysis } & \multicolumn{3}{|c|}{ Multivariate analysis } \\
\hline & OR & $95 \% \mathrm{Cl}$ & $p$ value & OR & $95 \% \mathrm{Cl}$ & $p$ value \\
\hline Age & & & & 1.52 & $0.98-2.32$ & 0.05 \\
\hline Age & & & & 1.41 & $0.95-2.05$ & 0.08 \\
\hline Frontal lobe (left) & 1.11 & $1.00-1.22$ & 0.05 & 1.11 & $0.99-1.26$ & 0.07 \\
\hline Medial frontal gyri & 1.05 & $1.00-1.09$ & 0.05 & 1.07 & $0.99-1.15$ & 0.06 \\
\hline
\end{tabular}

$\mathrm{Cl}$ - confidence interval; OR — odds ratio

occipital lobe [18] and reduced CBF in the occipital lobe, along with a relative increase in CBF in the deep white matter [19]. However, the actual CBF varies from reduced to normal in the frontal, parietal, temporal, and occipital lobes and in the basal ganglia [20]. In this study, we found reduced CBF in the orbital gyri, an area where reduced CBF has been found to correlate with the severity of Alzheimer's disease [21], which is thought to be related to Yakovlev's circuit involving the amygdala-thalamus-orbital gyri-anterior temporal lobe areas [21, 22]. In addition, there is some supporting evidence that the volume of the medial temporal lobe is better maintained in patients with Lewy body disease than in those with Alzheimer's disease [14]. A statistical parametric mapping analysis found reduced $\mathrm{CBF}$ in the supplementary motor area and showed that the CBF in the dorsolateral premotor cortex decreases as the severity of the disease [20]. The differences between these studies are thought to be due to non-uniform areas of functional decline, owing to differing assessment methods and differing disease-progression patterns in the selected cases. Although various tracers have been developed for PD and DLB, tracers that specifically bind to alpha-synuclein protein aggregates could help clarify the pathologies of these conditions and provide data for assessing treatments and prognosis.

A greater distribution of Lewy bodies in the temporal lobes is associated with a higher frequency of visual hallucinations [23]. Pathological examination of the brains of deceased patients with PD or DLB and of those who experienced visual hallucinations has shown the presence of large numbers of Lewy bodies in areas of the temporal lobe, such as the amygdala and parahippocampal gyri [23] Furthermore, the presence of many Lewy bodies has been noted in the middle frontal, middle temporal gyri, transentorhinal, and anterior cingulate cortices of patients with visual hallucinations [24].

Moreover, visual hallucinations in patients with DLB have been linked to reduced CBF in the left ventral occipital gyrus and bilateral parietal areas [25]. In our study, compared to patients who did not experience visual hallucinations, patients with new-onset Lewy body disease who experienced visual hallucinations exhibited significantly lower CBF in the frontal lobe. In addition, the frontal lobe CBF was significantly lower in the medial prefrontal gyri in patients with visual hallucinations than in those without visual hallucinations, for reasons that are yet unclear. Reduced CBF affected the occipital lobe, temporal lobe, parietal lobe, and frontal lobe, in that order. These findings together with the link between Lewy bodies and visual hallucinations suggest that the presence of a large number of Lewy bodies in the frontal lobes may indirectly reflect the reduced frontal lobe CBF in patients with visual hallucinations. The appearance of visual hallucinations may be affected by reduced nerve function, not only in the occipital lobe but also in a wide area extending to the medial frontal area. The relationship between the reduced CBF in anterior brain regions and the higher brain dysfunction associated with these anterior regions needs to be clarified and the pathology of nerve fiber routes should be explored.

Z-scores used in this study were based on normal database values. The utility of Z-scores for ${ }^{23}$-IMP SPECT imaging in the differentiation between Alzheimer's disease (AD) and DLB and other dementia and degenerative changes has been reported [26]. Furthermore, in cases of Alzheimer's dementia, Z-scores were shown to correlate inversely with the Mini-Mental State Examination and the Clinical Global Impression-Severity IIIness scores [27]. Therefore, we believe our data are clinically meaningful.

Visual hallucinations are present in a substantial proportion of patients with $\mathrm{PD}$, reportedly affecting $15.8 \%$ to $74 \%$ of patients, including some with recent-onset PD [28-30]. Notably, the frequency of visual hallucinations was reported to be $44.4 \%$ in DLB [31]. The occurrence of visual hallucinations among patients with DLB is marked and outweighs that among patients with PD (62.5\% vs $20.0 \%$ [32]. In a recent meta-analysis, the pooled prevalence of visual hallucinations was shown to be $28.2 \%$ and $61.8 \%$ among patients with PD and those with DLB, respectively [33]. Thus, the extreme difference in the prevalence of visual hallucinations between our study patients with PD and those with DLB is not unusual. As visual hallucinations can appear early in patients with $\mathrm{PD}$, the occurrence of visual hallucinations is not relied upon for a differential diagnosis of DLB (vs PD). Nuclear imaging, however, appears to be reliable for distinguishing between the two diseases.

Our study was limited in that it was a single-center study that included a small number of patients. In addition, the disease had reached H\&Y stage III or IV in some patients by the time of the scintigraphic examination, despite the study only including patients with new-onset Lewy body disease. Some of the patients were older adults, and the ability of-these patients to perform activities of daily living was indeed impaired. Despite the mixed study group, our study showed that reduced CBF is observed in cases of DLB and in patients suffering hallucinations, regardless of the H\&Y stage. The manifestations of 
Lewy body disease vary depending on the version of the disease. There is the presence of heterogeneity and some subgroup variation in early-stage PD [34]. Multicenter prospective studies with larger numbers of patients are needed to confirm our findings.

\section{Conclusions}

In conclusion, the results of our study showed that the CBF is reduced in several areas of the cerebral cortex and suggest an association between reduced blood flow in the frontal lobe and the appearance of visual hallucinations in patients with new-onset Lewy body disease.

\section{Conflict of interest}

Yukinori Okada received the lecture fee from AstraZeneca and Beyer not applicable the COI value in Japan, and the authors of this work have nothing to disclose for this study.

\section{Acknowledgments}

The authors thank Dr. Tsutomu Kamo of the Noborito Neurology Clinic and Prof. Tina Tajima of the St. Marianna University School of Medicine for their valuable advice regarding the study design.

\section{References}

1. Dias JA, Felgueiras MM, Sanchez JP, et al. The prevalence of Parkinson's disease in Portugal. A population approach. Eur J Epidemiol. 1994; 10(6): 763-767, doi: 10.1007/BF01719295, indexed in Pubmed: 7672060.

2. Cheng HC, Ulane CM, Burke RE. Clinical progression in Parkinson disease and the neurobiology of axons. Ann Neurol. 2010; 67(6): 715-725, doi: 10.1002/ana.21995, indexed in Pubmed: 20517933.

3. Obeso JA, Stamelou M, Goetz CG, et al. Past, present, and future of Parkinson's disease: A special essay on the 200th Anniversary of the Shaking Palsy. Mov Disord. 2017; 32(9): 1264-1310, doi: 10.1002/mds.27115, indexed in Pubmed: 28887905

4. Barnes J, David AS. Visual hallucinations in Parkinson's disease: a review and phenomenological survey. J Neurol Neurosurg Psychiatry. 2001; 70(6): 727-733, doi: 10.1136/jnnp.70.6.727, indexed in Pubmed: 11385004.

5. Iaccarino L, Sala A, Caminiti SP, et al. The brain metabolic signature of visual hallucinations in dementia with Lewy bodies. Cortex. 2018; 108: 13-24, doi: 10.1016/j.cortex.2018.06.014, indexed in Pubmed: 30098477.

6. Imabayashi E, Soma T, Sone D, et al. Validation of the cingulate island sign with optimized ratios for discriminating dementia with Lewy bodies from Alzheimer's disease using brain perfusion SPECT. Ann Nucl Med. 2017; 31 (7): 536-543, doi: 10.1007/s12149-017-1181-4, indexed in Pubmed: 28547521.

7. Imon $\mathrm{Y}$, Matsuda $\mathrm{H}$, Ogawa M, et al. SPECT image analysis using statistical parametric mapping in patients with Parkinson's disease. J Nucl Med. 1999; 40(10): 1583-1589, indexed in Pubmed: 10520695.

8. Imamura T, Ishii K, Sasaki M, et al. Regional cerebral glucose metabolism in dementia with Lewy bodies and Alzheimer's disease: a comparative study using positron emission tomography. Neurosci Lett. 1997; 235(1-2): 49-52, doi: 10.1016/s0304-3940(97)00713-1, indexed in Pubmed: 9389593.

9. Imabayashi E, Yokoyama K, Tsukamoto T, et al. The cingulate island sign within early Alzheimer's disease-specific hypoperfusion volumes of interest is useful for differentiating Alzheimer's disease from dementia with Lewy bodies. EJNMMI Res. 2016; 6(1): 67, doi: 10.1186/s13550-016-0224-5, indexed in Pubmed: 27620458.
10. Minoshima S, Frey KA, Koeppe RA, et al. A diagnostic approach in Alzheimer's disease using three-dimensional stereotactic surface projections of fluorine-18-FDG PET. J Nucl Med. 1995; 36(7): 1238-1248, indexed in Pubmed: 7790950.

11. Yamazaki $T$, Inui $Y$, Ichihara $T$, et al. Clinical utility of the normal database of I-iodoamphetamine brain perfusion single photon emission computed tomography for statistical analysis using computed tomography-based attenuation correction: a multicenter study. Ann Nucl Med. 2019; 33(11): 835-841, doi: 10.1007/s12149-019-01395-0, indexed in Pubmed: 31414335.

12. Vander Borght T, Minoshima S, Giordani B, et al. Cerebral metabolic differences in Parkinson's and Alzheimer's diseases matched for dementia severity. J Nucl Med. 1997; 38(5): 797-802, indexed in Pubmed: 9170449.

13. Gibb WR, Lees AJ. The relevance of the Lewy body to the pathogenesis of idiopathic Parkinson's disease. J Neurol Neurosurg Psychiatry. 1988; 51(6): 745-752, doi: 10.1136/jnnp.51.6.745, indexed in Pubmed: 2841426.

14. McKeith IG, Dickson DW, Lowe J, et al. Consortium on DLB. Diagnosis and management of dementia with Lewy bodies: third report of the DLB Consortium. Neurology. 2005; 65(12): 1863-1872, doi: 10.1212/01. wnl.0000187889.17253.b1, indexed in Pubmed: 16237129.

15. Fahn S, Elton RL. Members of the UPDRS Development Committee. Unified Parkinson's Disease Rating Scale. In: Fahn S, Marsden CD, Goldstein M, Calne DB. ed. Recent Developments in Parkinson's Disease, Vol 2. Macmillan Healthcare Information, Florham Park, NJ 1987: 153-163.

16. Fällmar D, Lilja J, Danfors T, et al. Z-score maps from low-dose F-FDG PET of the brain in neurodegenerative dementia. Am J Nucl Med Mol Imaging. 2018; 8(4): 239-246, indexed in Pubmed: 30245916.

17. Pasquier J, Michel B, Brenot-Rossi I, et al. Value of 99mTc-ECD SPET for the diagnosis of dementia with Lewy bodies. Eur J Nucl Med Mol Imaging. 2002; 29(10): 1342-1348, doi: 10.1007/s00259-002-0919-x, indexed in Pubmed: 12271417.

18. Ishii K, Yamaji S, Kitagaki $\mathrm{H}$, et al. Regional cerebral blood flow difference between dementia with Lewy bodies and AD. Neurology. 1999; 53(2): 413-416, doi: 10.1212/wnl.53.2.413, indexed in Pubmed: 10430439

19. Shimizu S, Hanyu H, Hirao K, et al. Deep gray matter hyperperfusion with occipital hypoperfusion in dementia with Lewy bodies. Eur J Neurol. 2007; 14(11): 1299-1301, doi: 10.1111/j.1468-1331.2007.01951.x, indexed in Pubmed: 17877736.

20. Kikuchi A, Takeda A, Kimpara T, et al. Hypoperfusion in the supplementary motor area, dorsolateral prefrontal cortex and insular cortex in Parkinson's disease. J Neurol Sci. 2001; 193(1): 29-36, doi: 10.1016/s0022510x(01)00641-4, indexed in Pubmed: 11718747.

21. Kawasaki Y, Ohtaki J, Toba K, et al. Relationship of Alzheimer disease severity and 99mTc-ECD SPECT brain blood-flow imaging (in Japanese). J Kyorin Med Soc. 2007; 38: 21-28.

22. Livingston KE, Escobar A. Anatomical bias of the limbic system concept. A proposed reorientation. Arch Neurol. 1971; 24(1): 17-21, doi: 10.1001/archneur.1971.00480310045003, indexed in Pubmed: 5538598.

23. Harding AJ, Broe GA, Halliday GM. Visual hallucinations in Lewy body disease relate to Lewy bodies in the temporal lobe. Brain. 2002; 125(Pt 2): 391-403, doi: 10.1093/brain/awf033, indexed in Pubmed: 11844739.

24. Gallagher DA, Parkkinen L, O'Sullivan SS, et al. Testing an aetiological model of visual hallucinations in Parkinson's disease. Brain. 2011; 134(Pt 11): 3299-3309, doi: 10.1093/brain/awr225, indexed in Pubmed: 21921019.

25. Nagahama Y, Okina T, Suzuki N, et al. Neural correlates of psychotic symptoms in dementia with Lewy bodies. Brain. 2010; 133(Pt 2): 557-567, doi: 10.1093/brain/awp295, indexed in Pubmed: 19920063.

26. Ishii K, Ito K, Nakanishi A, et al. Computer-assisted system for diagnosing degenerative dementia using cerebral blood flow SPECT and 3D-SSP: a multicenter study. Jpn J Radiol. 2014; 32(7): 383-390, doi: 10.1007/s11604014-0329-6, indexed in Pubmed: 24838777.

27. Kirino E. Three-dimensional stereotactic surface projection in the statistical analysis of single photon emission computed tomography data 
for distinguishing between Alzheimer's disease and depression. World J Psychiatry. 2017; 7(2): 121-127, doi: 10.5498/wjp.v7.i2.121, indexed in Pubmed: 28713690.

28. Aarsland D, Larsen JP, Cummins JL, et al. Prevalence and clinical correlates of psychotic symptoms in Parkinson disease: a community-based study. Arch Neurol. 1999; 56(5): 595-601, doi: 10.1001/archneur.56.5.595, indexed in Pubmed: 10328255

29. Coelho M, Marti MJ, Tolosa E, et al. Late-stage Parkinson's disease: the Barcelona and Lisbon cohort. J Neurol. 2010; 257(9): 1524-1532, doi: 10.1007/s00415-010-5566-8, indexed in Pubmed: 20411272.

30. Hely MA, Reid WGJ, Adena MA, et al. The Sydney multicenter study of Parkinson's disease: the inevitability of dementia at 20 years. Mov Disord. 2008; 23(6): 837-844, doi: 10.1002/mds.21956, indexed in Pubmed: 18307261.
31. Matar E, Ehgoetz Martens KA, Halliday GM, et al. Clinical features of Lewy body dementia: insights into diagnosis and pathophysiology. J Neurol. 2020; 267(2) 380-389, doi: 10.1007/s00415-019-09583-8, indexed in Pubmed: 31650254.

32. Savica R, Grossardt BR, Bower JH, et al. Incidence of dementia with Lewy bodies and Parkinson disease dementia. JAMA Neurol. 2013; 70(11): 1396-1402, doi: 10.1001/jamaneurol.2013.3579, indexed in Pubmed: 24042491.

33. Eversfield CL, Orton LD. Auditory and visual hallucination prevalence in Parkinson's disease and dementia with Lewy bodies: a systematic review and meta-analysis. Psychol Med. 2019; 49(14): 2342-2353, doi: 10.1017/S0033291718003161, indexed in Pubmed: 30474581.

34. Szeto JYy, O'Callaghan C, Shine JM, et al. The relationships between mild cognitive impairment and phenotype in Parkinson's disease. NPJ Parkinsons Dis. 2015; 1: 15015, doi: 10.1038/npjparkd.2015.15, indexed in Pubmed: 28725684 